\title{
Idoneidad epistémica de la unidad de números positivos y negativos del libro de texto oficial de matemáticas de séptimo grado de la Secretaría de Educación de Honduras
}

\section{Información \\ de artículo:}

\author{
Levin Dasayev Mendoza Sánchez ${ }^{1}$ \\ Yefrin Ariel Ortiz Pagoaga ${ }^{2}$ \\ Gerardo Alfredo Pinto Murillo 3 \\ Ivy Lou Green Arrechavala ${ }^{4}$
}

\section{Recibido: 30/03/2021 \\ Aprobado: 30/05/2021}

\section{Palabras claves:}

Idoneidad Epistémica, Significado holístico o de referencia, significado pretendido, configuración de objetos primarios.

\section{Resumen}

El enfoque ontosemiótico es un marco teórico integrativo que provee de herramientas teóricas necesarias para el estudio de los fenómenos didácticos en el proceso de enseñanza- aprendizaje de las matemáticas. En este estudio se presenta los resultados de la utilización de la noción de idoneidad didáctica y específicamente de la idoneidad en la faceta epistémica. En este sentido, se identificaron fortalezas y debilidades de un libro de texto de matemática desde la perspectiva ontológica y semiótica. Además se provee de insumos necesarios para la mejora epistémica del libro de texto, sus configuraciones ontosemióticas (objetos primarios) y una valoración de los contenidos presentados por este.

\section{Epistemic suitability of the unit of positive and negative numbers of the official seventh grade mathematics textbook of the Ministry of Education of Honduras}

\section{Abstract}

The ontosemiotic approach is an integrative theoretical framework that provides the theoretical tools necessary for the study of didactic phenomena in the teaching-learning process of mathematics. This study presents the results of

\footnotetext{
1 Licenciado en Matemáticas. Profesor de Matemáticas Dowal School, levindasayev@gmail.com (iD): https://orcid.org/oooo-0003-3904-1304

2 Maestro de Educación Primaria. Profesor de la Escuela Normal Mixta Pedro Nufio. Email: ortizyefrin18@gmail.com (iD): https://orcid.org/oooo-0oo3-1937-948X

3 Licenciado en Matemáticas. Profesor de la Universidad Pedagógica Francisco Morazán. Email: pintomath2o@gmail.com (iD): https://orcid.org/oooo-0oo1-6341-5391

4 Doctora en Educación. Profesora de la Universidad Pedagógica Nacional Francisco Morazán. Email: seminariozo16ii@gmail. com - (D): https://orcid.org/oooo-0oo1-8579-4599
}

Mendoza-Sánchez, L., Ortíz-Pagoaga, Y., Pinto-Murillo, G. \& Green-Arrechavala, I. (2021). Idoneidad epistémica de la unidad de números positivos y negativos del libro de texto oficial de matemáticas de séptimo grado de la Secretaría de Educación de Honduras. Revista Electrónica de Conocimientos, Saberes y Prácticas, 4(1), 102-117. https://doi. org/10.5377/recsp.v4i1.12098 
the use of the notion of didactic suitability and specifically of suitability in the epistemic facet. In this sense, strengths and weaknesses of a mathematics textbook were identified from the ontological and semiotic perspective. In addition, the necessary inputs are provided for the epistemic improvement of the textbook, its ontosemiotic configurations (primary objects) and an assessment of the contents presented by it.

\section{Introducción}

Los libros oficiales de matemáticas de la Secretaría de Educación de Honduras fueron creados en el marco del Proyecto Mejoramiento de la Enseñanza Técnica en el Área de Matemáticas (PROMETAM) con el objetivo de mejorar la calidad de la enseñanza en la asignatura de matemáticas (INICE, 2008). Ferrero (2006) define los libros de texto como objetos de estudio en diversos campos como la historia, la sociología, la educación, la didáctica, entre otras tantas. García-Martín (2014) menciona que "no cabe duda que el libro de texto de matemáticas es el recurso principal para explicar las clases de matemáticas y hacer problemas y ejercicios, sino que además es el eje vertebrador de la asignatura a lo largo del curso" (p. 13).

En Honduras Cárcamo (2012) estudio el uso de los libros por parte de los profesores, pero no se han realizado investigaciones que busquen analizar el libro de texto. Es en este sentido que nos preguntamos ¿Cuál es el grado de idoneidad epistémica que presenta la unidad de números positivos y negativos del libro de texto oficial de matemática de séptimo grado desde el punto de vista ontológico-semiótico? Usando el EOS para análisis de libros de textos se destacan la tesis de Gea Serrano (2014) y el estudio realizado a los libros de tres universidades por Acuña y Veloz (2017).

\section{Literatura}

Una de las novedades en este siglo, en cuanto a los enfoques teóricos en la educación matemática que sirven como base para la realización de investigaciones en este campo es el enfoque ontosemiótico (EOS) desarrollado por Godino y Batanero (1994); Godino, Batanero y Font (2008), entre otros. El enfoque ontosemiótico es un marco teórico integrativo que provee de herramientas teóricas necesarias para el estudio de las fenómenos didácticos en el proceso de enseñanza- aprendizaje de las matemáticas (Godino, 2017). Algunos de los conceptos del EOS que se utilizan en esta investigación se mencionan a continuación. Godino y Batanero (como se citó en Godino et al., 2006) definen significado como "los sistemas de prácticas operativas y discursivas que se ponen en juego por una persona (o compartidas en el seno de una institución) para resolver una cierta clase de situaciones - problemas". (p. 340). Godino et al. (2008) mencionan dos clases de significados, a saber, los significados institucionales y los significados personales los cuales se presentan en la tipología de la figura 1. 


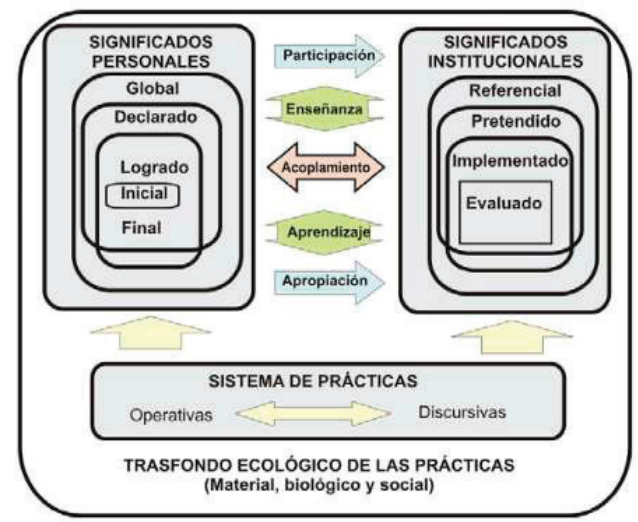

Figura 1: Tipos de significados institucionales y personales (Godino et al., 2008)

Otro concepto importante es el de objeto matemático para Godino et al. (2008) los objetos matemáticos se consideran entidades primarias, es decir elementos básicos de las prácticas matemáticas, que entran en acción al realizar e interpretar alguna tarea matemática determinada, es decir, "cuando un agente realiza y evalúa una práctica matemática activa un conglomerado formado por situaciones problemas, lenguajes, conceptos, proposiciones, procedimientos y argumentos" (p. 6). Estos objetos se relacionan entre si formando configuraciones las que llamamos configuraciones de objetos primarios o epistémicas. También definiremos análisis didáctico de acuerdo con Godino et al. (2006) como "el estudio sistémico de los factores que condicionan los procesos de enseñanza y aprendizaje de un contenido curricular - o de aspectos parciales del mismo-con unas herramientas teóricas o metodológicas específicas" (p. 4). El EOS propone cinco niveles entre los cuales la valoración de la idoneidad didáctica es uno de ellos (Font, Planas y Godino, 2010).

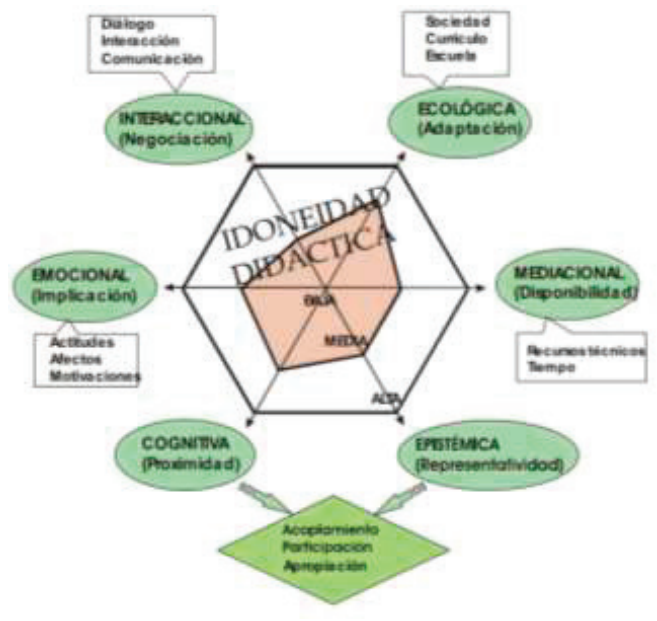

Figura 2: Componentes de la idoneidad didáctica (Godino, 2013, p.117) 
En el EOS idóneo se refiere a "el grado en que un proceso de estudio matemático (o una parte del mismo) permite el logro de los fines pretendidos" (Godino et al., 2006, p. 2). Por lo que la idoneidad didáctica se define como "criterio sistémico de pertinencia (adecuación al proyecto de enseñanza) de un proceso de instrucción” (Godino et al., 2006, p. 5). Esta idoneidad didáctica se divide en 6 facetas (Godino et al., 2006) que facilitan su análisis ya que de acuerdo con Godino et al. (2006) "La idoneidad didáctica supone la articulación coherente y armónica de las idoneidades parciales" (p. 5). En la figura 2 se pueden observar las componentes de la idoneidad didáctica Godino (2013) menciona que se sitúan en la base las idoneidad epistémica y la cognitiva ya que el proceso de estudio gira en torno a ciertos conocimientos dándoles un papel prioritario.

Por otro lado Godino (2013) menciona que los indicadores de idoneidad epistémica sirven para hacer operativa la noción de idoneidad didáctica estos componentes e indicares de la idoneidad epistémica se presentan en la tabla 1.

\begin{tabular}{|c|c|}
\hline \multicolumn{2}{|c|}{ Tabla 1: Componentes e indicadores de idoneidad epistémica } \\
\hline COMPONENTES & INDICADORES \\
\hline Situaciones- problemas & $\begin{array}{l}\text { - Se presenta una muestra representativa y ar- } \\
\text { ticulada de situaciones de contextualización, } \\
\text { ejercitación y aplicación. } \\
\text { - Se proponen situaciones de generación de prob- } \\
\text { lemas (problematización). }\end{array}$ \\
\hline Lenguajes & $\begin{array}{l}\text { - Uso de diferentes modos de expresión } \\
\text { matemática (verbal, gráfica, simbólica...), tra- } \\
\text { ducciones y conversiones entre los mismas. } \\
\text { - Nivel del lenguaje adecuado a los estudiantes a } \\
\text { que se dirige. } \\
\text { - Se proponen situaciones de expresión matemáti- } \\
\text { ca e interpretación. }\end{array}$ \\
\hline $\begin{array}{l}\text { Reglas (Definiciones, } \\
\text { proposiciones, proced- } \\
\text { imientos) }\end{array}$ & $\begin{array}{l}\text { - Las definiciones y procedimientos son claros y } \\
\text { correctos, y están adaptados al nivel educativo } \\
\text { al que se dirigen } \\
\text { - Se presentan los enunciados y procedimientos } \\
\text { fundamentales del tema para el nivel educativo } \\
\text { dado. } \\
\text { - Se proponen situaciones donde los alumnos ten- } \\
\text { gan que generar o negociar definiciones proposi- } \\
\text { ciones o procedimientos. }\end{array}$ \\
\hline Argumentos & $\begin{array}{l}\text { - Las explicaciones, comprobaciones y demostra- } \\
\text { ciones son adecuadas al nivel educativo a que se } \\
\text { dirigen. } \\
\text { - Se promueven situaciones donde el alumno } \\
\text { tenga que argumentar. }\end{array}$ \\
\hline Relaciones & $\begin{array}{l}\text { Los objetos matemáticos (problemas, defini- } \\
\text { ciones, proposiciones, etc.) se relacionan y } \\
\text { conectan entre sí. } \\
\text { - Se identifican y articulan los diversos significa- } \\
\text { dos de los objetos que intervienen en las prácti- } \\
\text { cas. }\end{array}$ \\
\hline
\end{tabular}




\section{Metodología de la Investigación}

El presente trabajo se enmarca en el enfoque cualitativo y es de tipo hermenéutico- dialéctico, para el cual se selección la unidad 1: "Números positivos y negativos" del libro de texto oficial de matemáticas de séptimo grado. La metodología que se utilizó se basó en lo propuesto por Pino-Fan, Castro, Godino y Font (2013) en la cual se mencionan tres etapas en la determinación de la idoneidad epistémica: 1. Determinar un significado global de referencia para los números positivos y negativos mediante una revisión de tipo histórico-documental. 2. Determinar el significado pretendido por la unidad de números positivos y negativo del libro de texto oficial de matemática. 3. Aplicar los criterios de idoneidad epistémica para valorar dicho libro de texto. Los instrumentos utilizados para recolectar la información son en primera instancia un cuestionario de lectura dirigida que constaba de 18 ítems divididos en los elementos de la configuración epistémica: conceptos, proposiciones, procedimientos, argumentos, situaciones-problema y elementos lingüísticos; en segundo lugar utilizamos una lista de cotejo con 29 ítems basados en los componentes de la idoneidad epistémica y sus indicadores: situaciones-problema, lenguaje, reglas, argumentos y relaciones. Para el análisis se hizo una comparación dialéctica entre los significados pretendido y referencial tomando como base los indicadores de idoneidad epistémica propuestos por Godino (2013).

\section{Resultados}

A continuación se presentan los resultados obtenidos durante la investigación, en primer lugar se hizo un análisis estructural de la unidad del libro de texto, luego se definieron los significados de referencia y pretendido relacionados con la unidad en cuestión y finalmente se valoró la idoneidad epistémica.

\subsection{Análisis estructural}

La Unidad 1: Números positivos y negativos está formada por tres lecciones en las cuales se presenta el contenido de la unidad además de un apartado de ejercicios y actividades de evaluación. En los apartados de ejercicios y evaluación se presentan situaciones relacionados con los temas de la unidad, ejercicios de práctica y problemas de aplicación. En la tabla 2 se resume la estructura de la unidad salta a la vista la forma en que están distribuidas las secciones en las lecciones del libro en las dos primeras lecciones la relación es de 4 a 5 secciones pero la lección 3 posee 12 secciones esto es una inconsistencia en la estructura del libro porque esta lección está subdivida en demasiadas partes y además la temática general, multiplicación y división de números positivos y negativos, no abarca los tópicos relacionados con las secciones 9 a la 12 esto es claramente un conflicto entre las conexiones de los distintos objetos matemáticos. 


\begin{tabular}{|c|c|c|}
\hline UNIDAD & LECCIÓN & SECCIÓN \\
\hline \multirow{23}{*}{$\begin{array}{l}\text { 1. Números positivos y } \\
\text { negativos }\end{array}$} & \multirow{4}{*}{ 1. Números positivos y negativos } & 1.Uso de los números positivos y negativos \\
\hline & & 2. Representación gráa \\
\hline & & 3. Relación de orden \\
\hline & & 4. Valor Absoluto \\
\hline & \multirow{5}{*}{$\begin{array}{l}\text { 2. Adición y sustracción de números positivos } \\
\text { y negativos }\end{array}$} & 1. Adición de números con igual signo \\
\hline & & 2. Adición de números con diferente signo \\
\hline & & 3. Propiedad conmutativa y asociativa de la adición \\
\hline & & 4. Sustracción \\
\hline & & 5. Planteamiento solo con adición \\
\hline & \multirow{12}{*}{$\begin{array}{l}\text { 3. Multiplicación y división de números } \\
\text { positivos y negativos }\end{array}$} & 1. Multiplicación \\
\hline & & 2. Propiedad conmutativa y asociativa de la multiplicación \\
\hline & & 3. División \\
\hline & & 4. Conversión de fracciones a decimales \\
\hline & & 5. Reciproco o inverso multiplicativo \\
\hline & & 6. Fracción compleja \\
\hline & & 7. Potencias y raíces \\
\hline & & 8. Exponente negativo \\
\hline & & 9. Adición y sustracción combinadas \\
\hline & & 10. Operaciones combinadas \\
\hline & & 11. Propiedad distributiva \\
\hline & & 12. Aplicación de los números negativos \\
\hline & \multirow{2}{*}{ Ejercicios y evaluación } & Ejercicios \\
\hline & & Evaluación \\
\hline
\end{tabular}

En relación al apartado de ejercicios y evaluación es necesario recalcar la cantidad de actividades que presenta en las que toma en cuenta temas de las lecciones que componen la unidad con el fin de que los estudiantes repasen los procedimientos y conceptos matemáticos trascendentales de la unidad esto demuestra que cumple con el indicador de situaciones- problema al presentar una buena muestra de actividades de ejercitación. Otra cosa interesante que presenta este apartado es la sección de evaluación en la cual los autores del libro proponen una serie de preguntas que se responde conociendo las definiciones, teoremas o proposiciones presentes en la unidad estos aspectos son positivos ya que demuestran buena relación entre los temas y las actividad de refuerzo y práctica y además suponer la necesidad de argumentar.

El libro utiliza un conjunto de símbolos que subdividen cada una de las partes de las secciones, estos símbolos se obtuvieron del libro del estudiante (Matemáticas 7 Libro del Estudiante) y de la guía del docente (Matemáticas 7 Guía del Docente) se obtuvieron los nombres de los mismos las se hace una descripción de los mismos (tabla 3) en relación al uso que observado de estos símbolos a los largo de la unidad de números positivos y negativos. 


\begin{tabular}{|c|c|c|}
\hline Símbolo & Objeto & Descripción \\
\hline 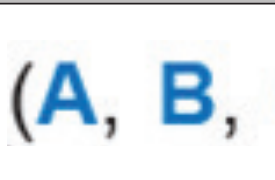 & Ejemplos de la sección & $\begin{array}{l}\text { Se utilizan las letras mayúsculas en color azul acom- } \\
\text { pañadas de un número para definir los ejemplos del } \\
\text { libro y los problemas introductorios (A1, A2, B1, B2, } \\
\text { etc.) }\end{array}$ \\
\hline & Respuestas a los ejemplos & $\begin{array}{l}\text { Este símbolo determina la respuesta dada por el libro } \\
\text { de texto a las determinadas situaciones presentadas } \\
\text { como ejemplos en cada sección }\end{array}$ \\
\hline & Ejercicios & $\begin{array}{l}\text { Se presentan los ejercicios de práctica, problemas y } \\
\text { aplicación de cada una de las secciones. }\end{array}$ \\
\hline$(1),(2),(3), \ldots$ & $\begin{array}{l}\text { Incisos de los ejercicios y ejem- } \\
\text { plos }\end{array}$ & $\begin{array}{l}\text { Se usan para definir los incisos en que subdivide algún } \\
\text { problema, ejercicio, ejemplo o aplicación. Inclusive } \\
\text { siendo los números de color azul definen los casos que } \\
\text { puede tomar alguna situación. }\end{array}$ \\
\hline & Puntos importantes & $\begin{array}{l}\text { En estos apartados se presentan definiciones, propie- } \\
\text { dades y proposiciones relacionadas con las temáticas } \\
\text { desarrolladas en las secciones. }\end{array}$ \\
\hline & Explicaciones relevantes & $\begin{array}{l}\text { Este símbolo hace referencia principalmente a proce- } \\
\text { dimientos, teoremas e incluso a propiedades de los } \\
\text { objetos matemáticos con que se están trabajando. }\end{array}$ \\
\hline & Notas & $\begin{array}{l}\text { Usualmente este apartado menciona arreglos en la } \\
\text { notación matemática, reglas de escritura y algunos } \\
\text { teoremas. }\end{array}$ \\
\hline
\end{tabular}

En la presentación de los ejemplos la forma sugerida por la guía del docente es contrastante con lo que está escrito en el libro del estudiante ya que se entiende que se presentará siguiendo la secuencia $\mathrm{A}_{1}, \mathrm{~A}_{2}, \ldots, \mathrm{B}_{1}, \mathrm{~B}_{2}, \ldots$, pero en solo el primero de los ejemplos relacionados tiene la nomenclatura esperada (A1) y los demás se limitan a escribir solo el número del ejemplo en color azul (4), esto representa un conflicto relacional ya que el libro reutiliza ejemplos haciendo referencia a estos en secciones anteriores del libro el problema radica en que se llama a los mismos por la secuencia sugerida pero en el libro se presenta de forma distinta, para el caso en la sección 1 de la lección 2 se hace referencia al ejemplo A7 pero para localizarlo es necesario antes saber el número de la página, lo cual es innecesario y fuerza una referencia doble, por medio del número de página y por el número de ejemplo, esta referencia aparece insertada entre paréntesis en el texto como se hace notar en la figura 3. Cabe destacar que en los apartados explicaciones relevantes y puntos importantes mencionados en la tabla 3 no existe un acuerdo por parte de los autores en los objetos matemáticos 
que estos presentan ya que más allá de que en realidad presentan elementos exclusivos, por ejemplos los puntos importantes son los únicos que incluyen definiciones y las explicaciones relevantes son las únicas que diagraman procedimientos, ambos incluyen de forma indistinta propiedades. Por otro lado las notas también presentan ciertos conflictos la mayoría de estas aclaran detalles de diversa índole pero algunas hacen referencia a teoremas los cuales deberían estar incluidos en los puntos importantes, esto conflictos son de tipo estructural que aunque no inciden directamente en la idoneidad epistémica si restan claridad al libro de texto.

Sección t: Adición de números con igual signo

A1| Recordemos lo visto en A7 (Página 4) para analizar la situación que se presenta a continuación. Fijémonos en la dirección de los movimientos y en la posición fínal.

Figura 3: Ejemplo lección 2 (Matemáticas 7 Libro del Estudiante, p. 11)

La secuencia didáctica, la cual se refiere a las relaciones, que sigue cada lección en la presentación de los significados pretendidos se describió de la siguiente manera: en primer lugar las lecciones de esta unidad comienzan siempre con alguna situación problemática, en el caso de las lecciones 2 y 3 se utiliza algunos de los problemas presentados como ejemplo en la lección 1 pero brindando nuevas condiciones a los mismos, estas reformulación de los problemas permite utilizar contextos problemáticos familiares como base para el desarrollo de nuevos objetos matemáticos (definiciones, proposiciones, etc.). Luego del concepto central aparecen las propiedades, teoremas, representaciones y procedimientos llegando a otros conceptos consecuentes o relacionados, esto ocurre en las dos primeras lecciones pero en la lección 3 como ya hemos mencionado. Finalmente en cada sección se presentan algunas situaciones problemáticas, ejercicios y aplicaciones para afianzar los conceptos y procedimientos propuestos.

\subsection{Significado de referencia}

El concepto de número (positivo) como tal es parte de la historia de la humanidad todas las culturas poseen alguna idea en relación a los números ya sea haciendo muescas en una rama, usando cordeles como los Incas o llegando civilizaciones con sistemas de numeración bien definidos como los Egipcios, Sumerios, Griegos, Romanos, Mayas, etc. hasta llegar a nuestro sistema de numeración y el concepto moderno de número que poseemos. Godino, Font, Wilhelmi y Arreche (2009) presentan las diversidad de significados que este constructo posee dividiendo los mismos en empíricos y formales. En primer lugar los significados empíricos comienzan con la idea de conteo además mencionan los siguientes usos "Para comunicar a otras personas, y como medio de registrar para nosotros mismos en otros momentos, el tamaño o cantidad de elementos de un conjunto de objetos discretos" (p. 38). En segundo lugar menciona los significados formales que adquiere este constructo matemático desde los axiomas de 
Peano y culmina diciendo que fue hasta finales del siglo XIX que "se fundamenta toda la matemática sobre los números naturales" (p. 40).

Por otro lado, se define el significado de referencia en relación a los números negativos fundamentado en la investigación presentada por Gallardo y Basurto (2010) en donde desde una perspectiva histórico- matemática más que educativa se describe la evolución de la negatividad matemática en la historia de la humanidad. Gallardo y Basurto (2010) parafraseando a Lizcano (1994) mencionan que "la negatividad hace referencia a los antecedentes históricos de los números negativos" (p. 257) es por esto que este apartado utiliza esta categoría ya que se intenta describir el origen histórico de los números negativos. Estos autores definen tres episodios en el desarrollo de la negatividad “1. Nacimiento de la negatividad matemática, 2. Aparición de las soluciones negativas y 3. Surgimiento de la negatividad algebraico- geométrica" (p. 257) en cada una de estas etapas surgen culturas que dieron origen a este constructo matemático.

En la primera etapa aparece China en el año 480 A.C. donde se utiliza una especie de caja de valores para hacer cálculos con números y aparecen los números negativos como deuda, también aparecen ciertas reglas para la suma de números de igual signo y de distinto signo y la idea del cero. Pero se encuentra la idea de evitar las soluciones nulas y las negativas. En la segunda etapa se menciona a Nicolas Chuquet que en el apéndice de su obra llamada Marre publicada en 1881 expone nueve problemas que se consideraban como imposibles en su época porque sus soluciones eran negativas. En la tercera etapa se mencionan a Descartes y a Euler en los siguientes sentidos:

En el texto de Descartes el andamiaje geométrico vinculado al lenguaje algebraico le permitió admitir distintas formas de negatividad. Es notorio que Euler [necesitara] validar la multiplicación de cantidades opuestas, apoyándose en un recurso externo: la existencia de deudas. (p. 266).

Es a partir de esta información histórica que se deduce que la pluralidad de significados del concepto de número negativo se desarrolla de manera similar a la de número positivo pasando por las concepciones de las culturas en las que se encontraron con estos números hasta llegar a una formalización la cual ha sido muy reciente con el surgimiento del algebra abstracta.

\subsection{Significado Pretendido}

Con fines ilustrativos se presentan las configuraciones epistémicas de las tres lecciones que conforman la unidad y de esa manera pormenorizar los objetos primarios que estas contienen. La lección 1: Números positivos y negativos presenta la introducción del concepto de números con signo mediante la resolución de problemas en diversidad de contextos: temperatura, altura sobre el nivel del mar y prolongación este- oeste y norte- sur lo cual es positivo para el libro desde el punto de vista epistémico 
ya que propone diversidad de problemas, ejercicios y aplicaciones. Además el nivel de los problemas presentados es adecuado para el nivel de los estudiantes y los contextos de los mismos son familiares. Por otro lado esta lección presenta diversidad de representaciones de las ideas matemáticas por ejemplo la representación gráfica de los números positivos y negativos como se puede observar en la sección 2, también se proponen situaciones en la cuales se deba pasar de la representación simbólica $(+3,-5,+0.8, \ldots)$ a la notación gráfica y viceversa como los ejercicios 8 y 9 propuestos en la página 7. La configuración epistémica de la lección 1 se presenta en la figura 4.

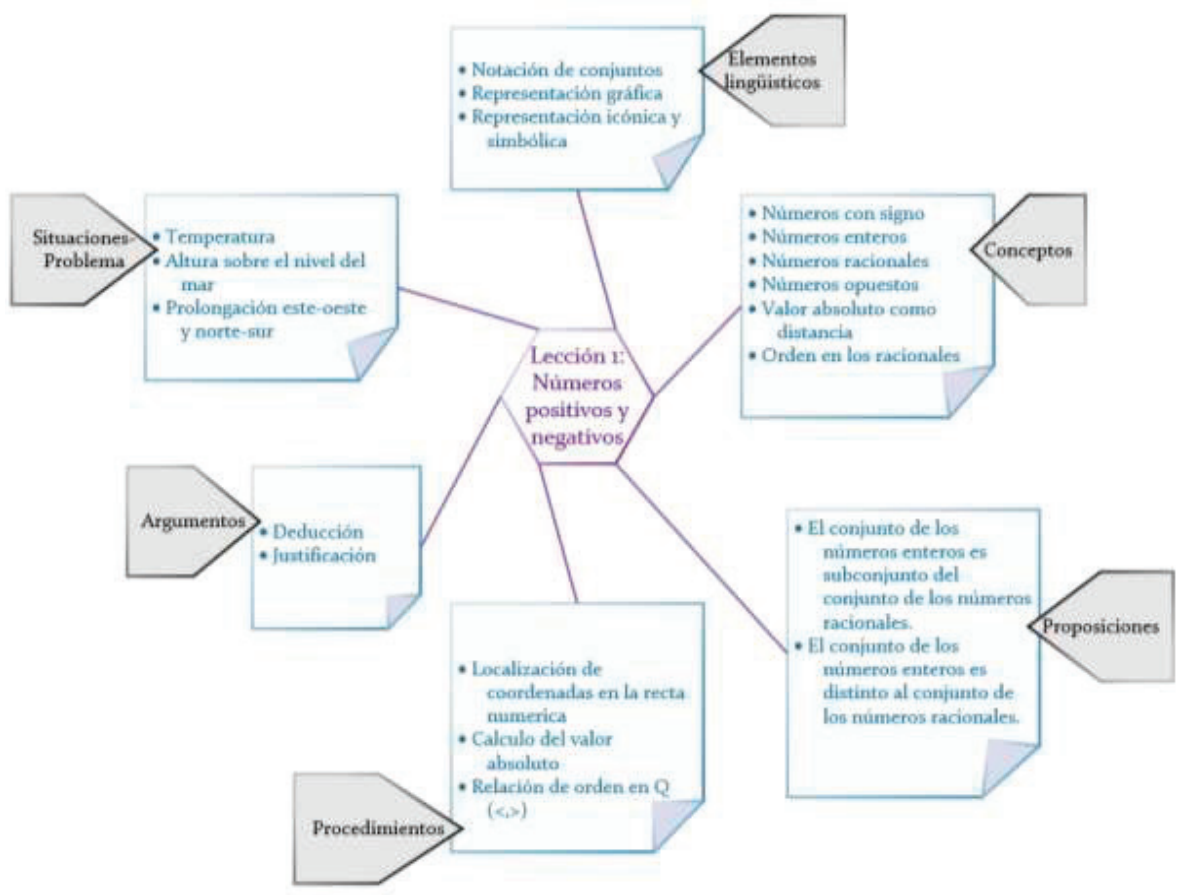

Figura 4: Configuración epistémica lección 1 (realización propia)

En la lección 2 Adición y sustracción de números positivos y negativos se hace uso de los problemas de la lección 1 pero además se proponen problemas de contexto netamente matemático para la comprobación de algunas propiedades de la suma esto se evidencia en la sección 3 de esta lección. En cuestión a la representación el libro realiza una adecuación curricular bastante interesante para expresar cantidades generales, como hasta este momento no se ha desarrollado el concepto de variable los autores optan por utilizar figuras geométricas en blanco como óvalos, rectángulos y triángulos que hacen las veces de números lo cual permite que los estudiantes escriban 
dentro de estas figuras o reemplacen las mismas con algún numero para corroborar las propiedades, un tipo de representación cuasi simbólica por ejemplo la propiedad asociativa de la página 14 del libro de texto. En la configuración epistémica para esta lección se observa que existen elementos comunes entre las configuraciones de las tres lecciones esto debido a la secuencia y diseño que sigue el libro, las principales similitudes se encuentran en las situaciones-problemas y en los argumentos utilizados para validar las ideas del libro. La lección 3 Multiplicación y división de números positivos y negativos es la más diversas de las tres lecciones llama la atención la inclusión de una sección completa de problemas de aplicación (sección 12) las situaciones- problemas presentados son muy diversos van desde pesos hasta llenado de tanques, por otro lado la cantidad de conceptos y propiedades matemáticas es impresionante esta lección es bastante formal sin alejarse de aquello que los estudiantes pueden comprender y las relaciones están bien definidas no hay saltos entre conceptos y lo que se usa ya ha sido previamente definido.

\subsection{Valoración de la idoneidad epistémica}

Luego de presentar los significados pretendidos y de referencia de la unidad de números positivos y negativos del libro de texto de matemáticas de séptimo grado se usaron los indicadores de idoneidad epistémica para valorar la idoneidad de la unidad, los datos recogidos usando estos indicadores, cada ítem puede tomar únicamente los valores cero o uno; el cero implica un fallo en relación a la presencia de dicho indicador en el texto o la presencia parcial del mismo, un uno significa la presencia de ese indicador en la unidad que estamos estudiando. Se presenta un promedio por cada ítem tomando en cuenta las tres lección y un promedio de la subcategoría de análisis este último promedio nos permite tener una idea de la idoneidad epistémica de la unidad completa. A primera vista se observan aquellas áreas en las cuales la unidad del libro de texto es bastante fuerte por ejemplo en situaciones- problema y en lenguajes el libro es bastante idóneo, en sentido opuesto la subcategoría menos idónea, en comparación con las otras es la de reglas pero de forma general está en un nivel medio-alto un resumen gráfico de lo que los indicadores de idoneidad epistémica nos dicen de la unidad se puede observar en la figura 5.

El pentágono regular supondría una idoneidad alta en cada una de las subcategorías que la componen mientas que el pentágono irregular nos muestra el nivel de idoneidad de cada categoría alcanzado por la unidad de nuestro análisis de forma general se observa una idoneidad media-alta por lo que podemos decir que el libro de texto es epistémicamente muy bueno. Una de las más grandes debilidades de la unidad del libro es el hecho de que no presenta situaciones en las cuales los estudiantes generar o crear procedimientos, esto tiene relación con la resolución de problemas que estén relacionados con las temáticas pero cuyas soluciones no se presenten de forma directa, quizá la razón para no presentar situaciones de esta clase es la dificultad implícita, pero se necesitan las mismas para desarrollar las habilidades de matematización 
mencionados en Godino (2013). Por otro lado y relación con la habilidad de argumentación la unidad propone pocas situaciones en las que se deban expresar ideas o interpretaciones para alcanzar una idoneidad epistémica mayor se deben incluir actividades de comunicación.

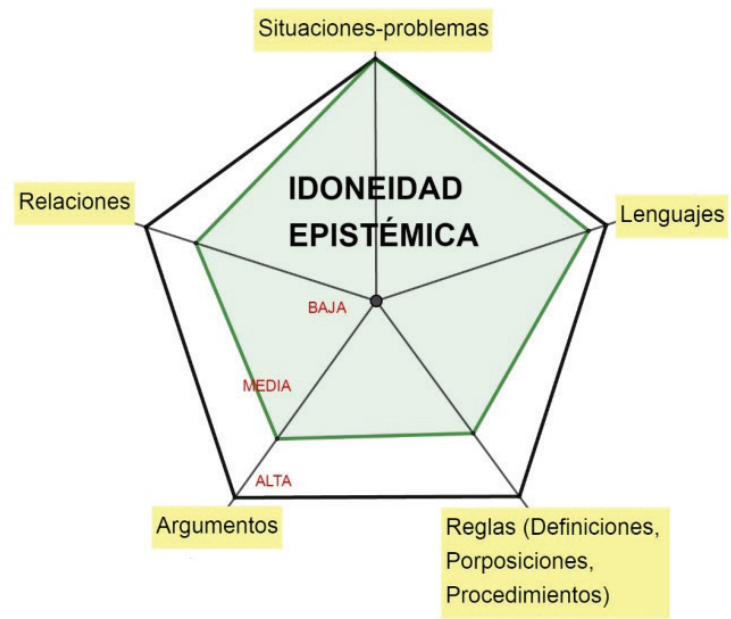

Figura 5: Idoneidad epistémica. Unidad 1 Números positivos y negativos (realización propia)

\subsection{Conflictos epistémicos encontrados}

La primera incidencia se dio en unos de los puntos importantes de la página el cual se muestra en la figura 6 , la definición desde el punto de vista matemático es correcto, pero menciona los conceptos mayores que cero y menores que cero este es un problema porque el concepto de mayor y menor implica la relación de orden el cual es un tema posterior como se puede ver en la tabla 5 ese tema corresponde a la sección 3. Mencionar esa relación es un problema porque hasta ese momento los estudiantes no tienen las herramientas necesarias para determinar el orden en los números con signo, especialmente los negativos. Una posible solución, que está relacionada con los problemas ya desarrollados en el libro, es definir los números positivos como los que están después del cero y los negativos como los que están antes.

Números positivos son los números que son mayores que cero.

Números negativos son los números que son menores que cero.

El número cero no es positivo ni negativo.

Figura 6: Definición de números positivos y negativos (Matemáticas 7 Libro del estudiante, p. 5)

En la página 4 en otro de los puntos importantes se menciona el término "características contrarias" para referirse a los números opuestos el conflicto aquí radica en 
que en la sección de valor absoluto en una de las explicaciones relevantes de la página 9 se menciona el término "números opuestos" el libro está usando estos términos como sinónimos pero no ha hecho la aclaración de que lo son por lo que para el estudiante son objetos distintos con características similares. En la definición de los números racionales de la página 6 como se muestra en la figura 7 también encontramos algunos conflictos epistémicos el libro sugiere que el denominador de un número racional es un número natural esto implica que los números como no son racionales allí surgen las preguntas si no son números racionales entonces ¿Qué son? Por otro lado y en la página 6 se define a los números decimales como "aquellos números racionales cuyos denominadores son potencias de 10" (p. 6), esta definición desde el punto de vista matemático es correcta pero puede presentar conflictos tomemos el caso del número racional según esta definición este número no es un número decimal porque el denominador no es una potencia de base 10, pero es cierto que es un número decimal periódico puro. De cualquier manera interpretando esta definición es posible decir que el es un número decimal si se escribe de la siguiente forma pero esto es un conflicto de Reglas ya que se deben presentar los conceptos de acuerdo al nivel al que se dirigen.

El conjunto de los números racionales se representa por la letra " $Q$ " y consiste en los números que se escriben de la forma racional, es decir, en la forma $\frac{\Delta}{\square}$ donde $\Delta$ es número enteroy $\square$ es un numero natural.

Figura 7: Definición de los números racionales (Matemáticas 7 Libro del estudiante, p. 6)

\section{Discusiones y Conclusiones}

De forma general la unidad 1 del libro de texto posee una idoneidad entre media y alta. Con respecto a la subcategoría de situaciones- problema posee una idoneidad alta desde el punto de vista de enfoque ontosemiótico esto es bueno ya que de acuerdo a Godino (2013) "se atribuye a las situaciones problemas un papel central, ya que se asume una concepción antropológica de la matemática, de modo que los objetos matemáticos emergen de las prácticas de los sujetos al enfrentarse a determinados problemas" (p. 9).

En el sentido de las representaciones de los constructos matemáticos el libro hace especial énfasis en la diversidad de los mismos proponiendo tareas que impliquen el cambio de una representación a otra, ya sea en lenguaje matemático o entre la lengua materna y el lenguaje matemático, esto es sinónimo de alta idoneidad epistémica y se puede observar en la valoración que se le brindó a la subcategoría de Lenguajes. Godino (2013) menciona en relación a las tareas propuestas por el libro que "deben proporcionar a los estudiantes diversas maneras de abordarlas, implicar diversas representaciones, y requerir que los estudiantes conjeturen, interpreten y justifiquen las soluciones" (p. 9). 
Más allá de que el libro presenta problemas en algunas definiciones, los conceptos matemáticos que intenta desarrollar están bien definidos y han sido adaptados curricularmente para la comprensión de los mismos por parte de los estudiantes, además los problemas propuestos para el desarrollo de los conceptos son idóneos para la creación de los objetos matemáticos por parte de los estudiantes. En este sentido Pino-Fan et al. (2013) citando a Godino y Batanero (1994) mencionan que "los significados logrados (aprendidos) por los estudiantes dependen fundamentalmente de los significados institucionales, concretamente, de los significados pretendidos asociados a los sistemas de prácticas planificados para un proceso particular de instrucción" (p. 147). En pocas palabras el libro posea una buena representatividad de los significados institucionales es de mucha ayuda para los docentes porque estos hacen uso los significados pretendidos para el logro de los mismo por parte de los estudiantes.

\section{En este sentido, se recomienda las apreciaciones siguientes:}

- La noción de idoneidad didáctica y más específicamente la noción de idoneidad epistémica es un conocimiento necesario de los profesores en formación así como para los profesores en ejercicio ya que de acuerdo a Godino et al. (2006) "Es necesario que los profesores planifiquen la enseñanza teniendo en cuenta los significados institucionales que se pretenden estudiar, adoptando para los mismos una visión amplia, no reducida a los aspectos discursivos (idoneidad epistémica)" (p. 22). Esto permite valorar la fidelidad de las pretensiones de los libros de texto y decidir que enseñar para alcanzar los objetivos educativos en el área de matemáticas.

- Se realizó una valoración de la idoneidad epistémica de parte del libro de texto, pero aún queda mucho por hacer, como por ejemplo se puede realizar las valoraciones de la idoneidad epistémica para los otros libros de la serie y para otros bloques de contenido (geometría, estadística, algebra) y así tener una idea de la idoneidad epistémica global de los libros de texto, también junto con esto puede hacerse una comparación entre los significados propuestos por los libros y los propuestos por el Diseño Curricular Nacional Básico. Por otro lado para valorar la idoneidad didáctica se pueden realizar investigaciones que complementan este estudio haciendo énfasis en las dimensiones mediacional y ecológica que de acuerdo con Godino (2013) y junto con la idoneidad epistémica son las esenciales para valorar la idoneidad didáctica global.

- Es necesario realizar otras investigaciones que utilicen el enfoque ontosemiótico en nuestro país, ya que es un marco referencial que integra diversas teorías y brinda perspectivas innovadoras a las situaciones que se presentan en los salones de clase de matemática, más allá de esta investigación no hay precedentes del uso de esta teoría en Honduras y teniendo en cuenta que somos un país que posee una universidad exclusiva para la formación de docentes es necesario estar al día con las tendencias investigativas en Educación Matemática para generar resultados que 
ayuden a la mejora de la enseñanza y aprendizaje de las matemáticas en nuestro contexto especifico.

\section{Lista de referencias}

Acuña, C, y Veloz, B. (2017). Análisis de los objetos matemáticos en libros de texto de ingeniería. En Contreras, J., Arteaga, P., Cañadas, G., Gea, M., Giacomone, B. y López, (Eds.), Actas del Segundo Congreso International Virtual sobre el Enfoque Ontosemiótico del Conocimiento y la Instrucción Matemáticos. Recuperado de: http://enfoqueontosemiotico.ugr.es/civeos/acu\%C3\%B1a_veloz.pdf

Cárcamo, D. (2012). Uso de los Libros de Texto de Matemática en el proceso de enseñanza: Un Análisis de casos comparado. (Tesis de maestría). Recuperado de http:// biblioteca.upnfm.edu.hn/images/tesis\%2oclasificadas/Maestria\%2oen\%20 formador\%2ode\%2oformadores\%20de\%2oeducacion\%2obasica/Donaldo\%20 Carcamo.pdf

Ferrero, S. A. (2006). La importancia de ser llamado "libro de texto." Hegemonía y control del currículo en el aula. Educación, Lenguaje YSociedad, 4(4), 181-189. Recuperado de http://web.a.ebscohost.com/ehost/pdfviewer/pdfviewer?vid=7\&sid=ef6de89122b7-4ac3-a849-e8647415e51c\%40sessionmgr4007

Font, V., Planas, N. y Godino, J. D. (2010). Modelo para el análisis didáctico en educación matemática. Infancia y Aprendizaje, 33 (2), 89-105. Recuperado de http:// www.ugr.es/ jgodino/eos/modelo_anadida_25junioog.pdf

Gallardo, A. y Basurto, E. (2010). La negatividad matemática: antesala histórica de los números enteros. Revista Latinoamericana de Investigación en Matemática Educativa, 13(4), 255-268. Recuperado de http://www.redalyc.org/pdf/335/33529137015.pdf

García Martín, A. (2014). El uso del libro de texto de matemáticas en el aula. Revisión del estado actual de la cuestión. Recuperado de http://hdl.handle.net/10481/36188

Gea Serrano, M. (2014). La correlación y regresión en bachillerato análisis de libros de texto y del conocimiento de los futuros profesores. (Tesis doctoral). Recuperado de http://hdl.handle.net/10481/34257

Godino, J. D. y Batanero, C. (1994). Significado institucional y personal de los objetos matemáticos. Recherches en Didactique des Mathématiques, 14 (3), 325355. Recuperado de http://www.ugr.es/ jgodino/funciones-semioticas/o3_ SignificadosIP_RDM94.pdf 
Godino, J. (2013). Indicadores de la idoneidad didáctica de procesos de enseñanza y aprendizaje de las matemáticas. Cuadernos de Investigación y Formación en Educación Matemática, 1(11), 111-132. Recuperado de http://revistas.ucr.ac.cr/ index.php/cifem/article/viewFile/14720/13965

Godino, J. (Productor). (2017). Presentación EOS [youtube]. De https://www.youtube. $\mathrm{com} /$ watch?v=UCsUuroPIQY

Godino, J., Batanero, C. y Font, V. (2008). Un enfoque ontosemiótico del conocimiento y la instrucción matemática. Acta Scientiae. Revista de Ensino de Ciências e Matemática, 10, 7-37. Recuperado de http://www.ugr.es/ jgodino/funcionessemioticas/sintesis_eos_1omarzoo8.pdf

Godino, J., Bencomo, D., Font, V. y Wilhelmi, M. R. (2006). Análisis y valoración de la idoneidad didáctica de procesos de estudio de las matemáticas. Paradigma, 27(2), 221-252. Recuperado de:http://www.ugr.es/ jgodino/funciones-semioticas/ idoneidad-didactica.pdf

Godino, J., Font, V., Wilhelmi, M. y Arreche, M. (2009). ¿Alguien sabe qué es el número? Unión 1(19), 34-46. Recuperado de http://www.fisem.org/www/union/ revistas/2009/19/Union_019_008.pdf

INICE Instituto nacional de investigación y capacitación educativa. (2008). Proyecto Regional iMe gusta Matemática! Proyecto Mejoramiento en la Enseñanza Técnica en el Área de Matemáticas (PROMETAM) Fase II.

Pino-Fan, L., Castro, W., Godino, J. y Font, V. (2013). Idoneidad epistémica del significado de la derivada en el currículo de bachillerato. Paradigma, 34(2), 129-150. Recuperado de http://www.scielo.org.ve/scielo.php?script=sci_ arttext\&pid=S1011-22512013000200008\&lng=es\&tlng=es. 